\title{
EXISTENCE AND COMPARISON OF EIGENVALUES OF $n$ TH ORDER LINEAR DIFFERENTIAL EQUATIONS
}

\author{
BY R. D. GENTRY ${ }^{1}$ AND C. C. TRAVIS
}

Communicated by Hans Weinberger, May 1, 1975

Consider the differential equations

$$
\left[a(x) u^{(k)}(x)\right]^{(n-k)}-(-1)^{n-k} \lambda \sum_{i=0}^{k-1} p_{i}(x) u^{(i)}(x)=0
$$

and

$$
\left[A(x) v^{(k)}(x)\right]^{(n-k)}-(-1)^{n-k} \Lambda \sum_{i=0}^{k-1} q_{i}(x) v^{(i)}(x)=0,
$$

subject to the boundary conditions

or

$$
\begin{aligned}
u(\alpha) & =u^{\prime}(\alpha)=\cdots=u^{(k-1)}(\alpha) \\
& =u_{1}(\beta)=u_{1}^{\prime}(\beta)=\cdots=u_{1}^{(n-k-1)}(\beta)=0, \\
v(\alpha) & =v^{\prime}(\alpha)=\cdots=v^{(k-1)}(\alpha) \\
& =v_{1}(\beta)=v_{1}^{\prime}(\beta)=\cdots=v_{1}^{(n-k-1)}(\beta)=0,
\end{aligned}
$$

$$
\begin{aligned}
u(\alpha) & =u^{\prime}(\alpha)=\cdots=u^{(k-1)}(\alpha) \\
& =u(\beta)=u^{\prime}(\beta)=\cdots=u^{(n-k-1)}(\beta)=0, \\
v(\alpha)= & v^{\prime}(\alpha)=\cdots=v^{(k-1)}(\alpha) \\
= & v(\beta)=v^{\prime}(\beta)=\cdots=v^{(n-k-1)}(\beta)=0,
\end{aligned}
$$

where $u_{1}(x) \equiv a(x) u^{(k)}(x)$ and $v_{1}(x) \equiv A(x) v^{(k)}(x)$. We assume that the functions $a(x), p_{0}(x)$, and $q_{0}(x)$ are positive on $[\alpha, \beta]$. Equations (1.1)-(1.2) subject to the boundary conditions (1.1a)-(1.2a) [(1.2b)-(1.2b), respectively] will be termed the $(k, n-k)$-focal point eigenvalue problem [the $(k, n-k)$-conjugate point eigenvalue problem, respectively].

We establish the existence of a smallest positive eigenvalue for both of these eigenvalue problems and prove comparison theorems relating the eigenvalues. Our

AMS (MOS) subject classifications (1970). Primary 34B05; Secondary 34C10.

Key words and phrases. $n$th order differential equation, eigenvalue, focal point, conjugate point, $u_{0}$-positive, positive cone.

${ }^{1}$ Supported by the National Council of Canada Grant A9100. 
results generalize the classical selfadjoint eigenvalue comparison theorems in two distinct ways. First, we allow the principal part of the differential equation to be of arbitrary order; thereby, allowing the problem to be nonselfadjoint. Second, even in the case where the principal part of the differential equation is formally selfadjoint, that is, when $n=2 k$, the eigenvalue problem does not reduce to a selfadjoint eigenvalue problem unless $p_{i}(x) \equiv 0$ for $i=1,2, \ldots, k-1$. Only in this case does the Courant Minimum Principle apply yielding that the last positive eigenvalues of (1.1) and (1.2) satisfy $\Lambda_{0} \leqslant \lambda_{0}$, if $0<A(x) \leqslant a(x)$ and $p_{0}(x) \leqslant$ $q_{0}(x)$ on $[\alpha, \beta]$. The following are an example of the type of results we have obtained for equations (1.1) and (1.2).

THEOREM 1.1. Assume that $0 \leqslant \int_{x}^{\beta} p_{i}(s) d s$ on $[\alpha, \beta]$ for $i=0,1, \ldots$, $k-1 ;\left[0=p_{i}(x)\right.$ on $[\alpha, \beta]$ for $i=1, \ldots, k-1$, respectively $]$; then the $(k, n-k)$-focal point eigenvalue problem [the $(k, n-k)$-conjugate point eigenvalue problem, respectively] has at least one real eigenvalue which is positive and smaller than the absolute value of any other eigenvalue. The eigenfunction associated with this eigenvalue is positive on $(\alpha, \beta)$.

THEOREM 1.2. If

$$
\begin{aligned}
& 0 \leqslant p_{i}(x) \text { on }[\alpha, \beta] \text { for } i=0, \ldots, k-1, \\
& \int_{x}^{\beta} p_{i}(s) d s \leqslant \int_{x}^{\beta} q_{i}(s) d s \quad \text { on }[\alpha, \beta] \text { for } i=0,1, \ldots, k-1, \\
& \int_{a}^{x} a(s) d s \leqslant \int_{\alpha}^{x} A(s) d s \text { on }[\alpha, \beta],
\end{aligned}
$$

then the smallest positive eigenvalues $\lambda_{0}$ and $\Lambda_{0}$ of the $(k, n-k)$-focal point eigenvalue problems (1.1) and (1.2), respectively, satisfy, $\Lambda_{0} \leqslant \lambda_{0}$ with equality if and only if $a(x) \equiv A(x)$ and $p_{i}(x) \equiv q_{i}(x)$ on $[\alpha, \beta]$ for $i=0,1, \ldots, k-1$.

Eigenvalue comparison theorems of the "integral type", such as appear in Theorem 1.2, were first established by Z. Nehari [1] for second order differential equations, and later extended to selfadjoint equations of order $2 n$ by C. C. Travis [2].

THEOREM 1.3. If

$$
\begin{gathered}
0<p_{0}(x) \leqslant q_{0}(x), \\
0=p_{i}(x)=q_{i}(x) \quad \text { on }[\alpha, \beta] \text { for } i=1,2, \ldots, k-1, \\
0<A(x) \leqslant a(x) \text { on }[\alpha, \beta],
\end{gathered}
$$

then the smallest positive eigenvalues $\lambda_{0}$ and $\Lambda_{0}$ of the $(k, n-k)$-conjugate point eigenvalue problems (1.1) and (1.2), respectively, satisfy $\Lambda_{0} \leqslant \lambda_{0}$ with equality if and only if $a(x) \equiv A(x)$ and $p_{0}(x) \equiv q_{0}(x)$ on $[\alpha, \beta]$.

Proofs and applications of the above results will appear elsewhere. 


\section{REFERENCES}

1. Z. Nehari, Oscillation criteria for second order linear differential equations, Trans. Amer. Math. Soc. 85 (1957), 428-445. MR 19, 415.

2. C. C. Travis, Comparison of eigenvalues for linear differential equations of order 2n, Trans. Amer. Math. Soc. 177 (1973), 363-374. MR 47 \#356.

DEPARTMENT OF MATHEMATICS, UNIVERSITY OF GUELPH, GUELPH, ONTARIO, CANADA

DEPARTMENT OF MATHEMATICS, UNIVERSITY OF TENNESSEE, KNOXVILLE, TENNESSEE 37916 